\title{
Origine et devenir des produits phytosanitaires
}

\author{
Origin and Fate of pesticides
}

\author{
par M. Schiavon et C. Perrin-Ganier \\ Laboratoire Sols et Environnement, ENSAIA/INRA,
}

Both agricultural practices and biophysicochemical properties of soils and chemicals play a significant part in the fate of pesticides in the environment.

A few farming types occupy the major area of cultivated lands. Due to their particular efficiency, some active ingredients are systematically used to protect these crop systems. As a consequence, soil ability to degrade or retain these pesticides should be insufficient. However, excess amounts of pesticides in soil are not the only cause of pollution.

The biocidal activity of these chemicals implies that they are available for living organisms, so that they are not strongly retained to soil and that they can enter the soil solution. As a consequence, pesticides in solution are potentially carried away by water movement. Yet, retention of pesticides by soil components changes with time and should lead, at least temporally, to non-available residues. Pesticide residues strongly sorbed to soil could then move along with soil particles or be released when organic material is reorganized during humification processes. In that case, pesticide residues are liable to be responsible for non-point pollution.

Fate of pesticide in soil is also determined by biological decomposition. Degradation processes may lead to the disappearing of parent compound and to the formation of degradation products, which could temporally accumulate in soils. So, biodegradation may result in a more diversified environmental pollution.

\section{I $\square$ INTRODUCTION}

La prise de conscience de la nécessité de protéger les cultures est certainement simultanée à la naissance de l'agriculture. De nombreuses années ont été nécessaires pour que la chimie voit le jour et prenne le relais des prières, des exorcisations et de la sorcellerie. Le soufre, l'arsenic, la nicotine et le sulfate de fer figurent parmi les premiers produits phytosanitaires. Cependant, les progrès réels en matière de protection des cultures sont très récents. Ils débutent avec la mise œuvre d'une politique de développement de l'agriculture permettant de répondre aux besoins alimentaires d'une population à la démographie croissante. Ils sont aussi associés aux progrès de l'agrochimie [1].

Même si l'efficacité de certains produits est connue dès les années 1930-1940, dans la pratique, c'est vers les années 1960 que les agriculteurs s'initient à leur utilisation. Dès lors, malgré les réticences des adeptes de l'agriculture biologique, se mettait en place la protection chimique des cultures - chaque jour un peu plus performante à l'égard de la maîtrise des ravageurs, des maladies et des adventices - et une agression progressivement perceptible des agrosystèmes et de l'environnement en général.
En effet, l'emploi de ces produits, même bien contrôlé, s'accompagne d'une certaine dispersion de la pulvérisation par voie aérienne. Par ailleurs, lorsqu'il s'agit du traitement des parties aériennes des végétaux, peu de produit reste sur la cible ; l'essentiel parvient au sol, directement ou indirectement par les pluvio-lessivats. Ainsi, hormis les pertes lors de la pulvérisation, le devenir des produits phytosanitaires est régulé par les pratiques de protection des cultures et par différents processus intervenant au niveau du sol en fonction des conditions climatiques. L'analyse de l'état de pollution de l'air, du sol et tout particulièrement de l'eau constitue un bon moyen pour appréhender le devenir de ces produits.

\section{II — LE MARCHÉ DES PRODUITS PHYTOSANITAIRES ET LES PRATIQUES DE PROTECTION DES CULTURES}

Plusieurs causes sont à l'origine de la pollution de l'environnement par les produits phytosanitaires. En premier lieu cette pollution a pour source les 77000 tonnes utilisées annuellement en France (tableau 1). 
Tableau I : Tonnage des matières actives entrant dans la composition de spécialités utilisées en France (UIPP, 1997)

\begin{tabular}{|c|c|}
\hline Famille & Quantités utilisées (t/an) \\
\hline Insecticides $^{\text {Fongicides }}{ }^{\circ}$ & 6074 \\
Herbicides $^{*}$ & 31415 \\
Autres* $^{*}$ & 33576 \\
\hline
\end{tabular}

${ }^{\circ}$ excepté le soufre et le cuivre, ${ }^{*}$ nématicides, rodenticides, molluscicides, substances de croissance, ....

Même si l'agriculture n'utilise pas la totalité de ces produits (figure 1), ce sont ses pratiques qui sont perçues comme globalement responsables de la dégradation de la qualité de l'eau. En effet, les conséquences de cet emploi massif de produits phytosanitaires sont aggravées d'une part, par une utilisation non pas répartie sur l'ensemble des 30534000 ha de surfaces agricoles, mais focalisée sur certaines cultures, et d'autre part, par l'utilisation systématique de certains produits particulièrement performants (figure 2). Ainsi, certaines molécules comme l'atrazine, l'isoproturon, le tébutame... sont susceptibles d'exercer une forte pression sur l'environnement alors que d'autres n'auront qu'une action plus localisée.

La situation créée par l'emploi de l'atrazine est à cet égard exemplaire. La pollution généralisée de l'eau par ce produit est due au désherbage systématique du maïs par cette matière active mais également à la place du maïs dans l'assolement du territoire et surtout à sa monoculture, conduisant au retour annuel de l'atrazine sur les mêmes surfaces.

\section{III — LA RÉTENTION DES PRODUITS PHYTO- SANITAIRES ET LEUR DISPERSION}

Dans la mesure où les produits utilisés finissent par arriver au sol, le deuxième aspect à considérer concerne leur devenir dans ce milieu. Ainsi, la pollution de l'eau par les pesticides est liée, d'une part à la nature des interactions sols-produits et d'autre part à la capacité de dégradation des populations microbiennes du sol (figure 3). L'ensemble est sous la dépendance des conditions climatiques.

Pour pouvoir exercer un effet biocide (élimination des mauvaises herbes, de ravageurs, ...) ces produits doivent rester, au moins partiellement, biodisponibles, c'est-à-dire utilisables par l'organisme à détruire. Ainsi, l'énergie des diverses liaisons impliquées dans leur adsorption doit être

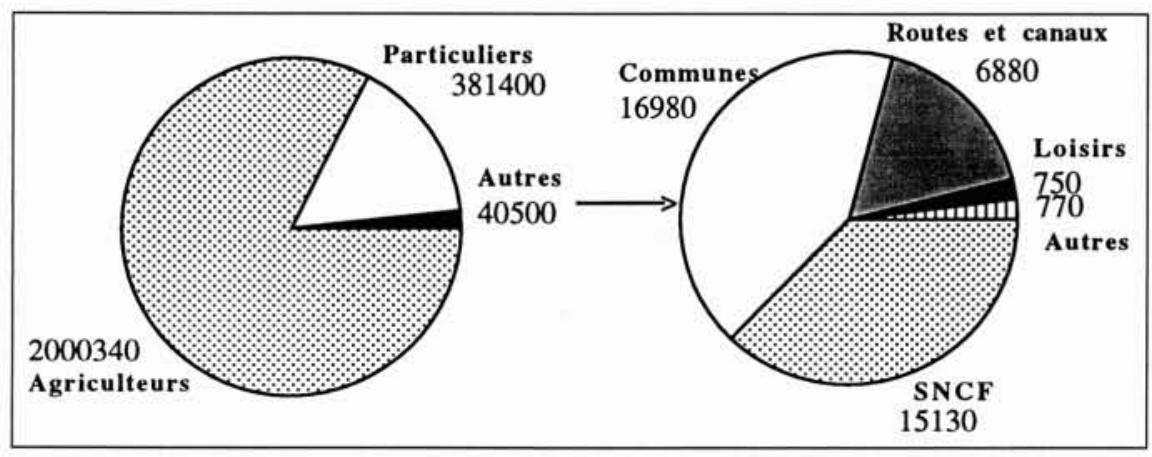

1. Utilisation des produits phytosanitaires en Lorraine. Valeurs données en kg. (d'après document DRAF/SRPV, 1996).

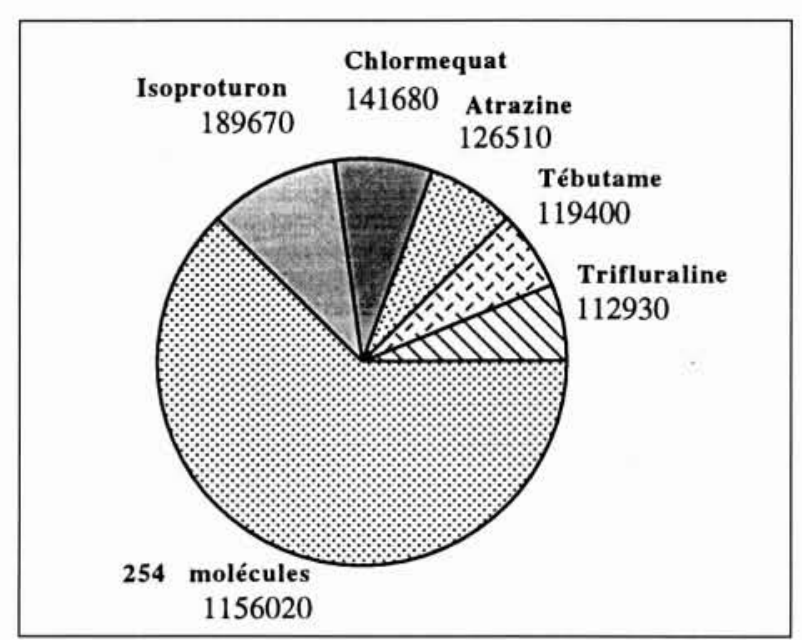

2. Les principales matières actives utilisées en agriculture en Lorraine. Valeurs données en $\mathrm{kg}$. (d'après document DRAF/SRPV, données 1996).

suffisamment faible pour permettre leur désorption et leur passage dans l'air ou dans la solution du sol sous forme dissoute. Ces produits sont généralement peu volatiles, c'est donc la phase aqueuse du sol qui représente le compartiment biodisponible prédominant. L'équilibre qui s'installe entre la quantité de produit dans la solution du sol et celle retenue par les constituants solides, défini par un coefficient de partage $(\mathrm{Kd})$, va dépendre de la taille respective des deux compartiments, de leurs propriétés physico-chimiques et de celles du produit phytosanitaire. Plus la valeur du Kd est faible et moins le produit sera retenu par les constituants du sol. Les produits polaires et/ou très hydrosolubles sont en général peu retenus par le sol et sont susceptibles d'être aisément dispersés.

Il convient de noter que la position des résidus sur les surfaces adsorbantes ainsi que la nature et l'énergie des liaisons qui s'établissent entre le sol et le pesticide sont susceptibles d'évoluer avec le temps. On parle de vieillissement des résidus dans le sol, qui se traduit par une diminution de leur disponibilité à l'extraction et donc à l'entrainement par l'eau sous forme dissoute, mais favorise leur transport à l'état adsorbé. C'est vraisemblablement ce phénomène qui est en partie responsable de la formation des résidus non extractibles.

La dispersion par l'eau d'un produit phytosanitaire est donc dépendante de sa rétention, mais elle est également tributaire de la durée de vie du produit dans le sol. Ainsi, plus une molécule sera récalcitrante à la biodégradation et plus sa présence sera probable lors de l'arrivée au sol d'un excédent d'eau.

L'évolution des forces de rétention et la dégradation progressive des produits se traduit dans la pratique par une pollution d'autant plus aiguë de l'eau qui circule à la surface ou à travers le sol, que le délai entre le traitement et les précipitations qui donnent lieu à ces excédents d'eau est court (Babut et al., 1996 ; [3]) (tableau 2).

Par ailleurs, l'intensité des transferts est différente suivant les pro- 
priétés physiques des sols. En effet, pour les produits appliqués au printemps sur sols drainés, quelle que soit l'année, les concentrations maximales observées et les quantités globales transportées par les eaux de drainage sont régulièrement supérieures dans un sol argileux que dans un sol limoneux présentant un même $\mathrm{Kd}$. Ce comportement différent des produits dans les deux types de sol est à mettre en relation avec la structure obtenue pour chacun d'eux après la préparation du lit de semence : structure grossière et hétérogène pour les sols argileux, structure plus fine et plus homogène pour les sols limoneux. Ces différences de structure et donc de porosité modifient les interactions eau-pesticide et de ce fait le transfert.

Mais cette différence de comportement des produits en fonction du type de sol peut s'effacer. A l'automne ou en hiver, les sols perdent leur porosité grossière. Comme ils sont engorgés d'eau, un drainage quasi continu se met en place. Pour les produits appliqués à cette période, par exemple le chlortoluron, aucune différence significative de comportement n'est alors observée entre un sol argileux et un sol limoneux, et les quantités exportées sont étroitement corrélées aux volumes drainés (figure 4). Tout se passe comme si le transfert n'était plus régulé que par des processus d'adsorption-désorption sensiblement identiques quelles que soient les caractéristiques granulométriques des sols.

Enfin, la formation dans le sol de résidus fortement retenus ou non extractibles conduit à une pollution de l'eau faible mais prolongée dans le temps $[4,5,6,7]$. Ainsi, après

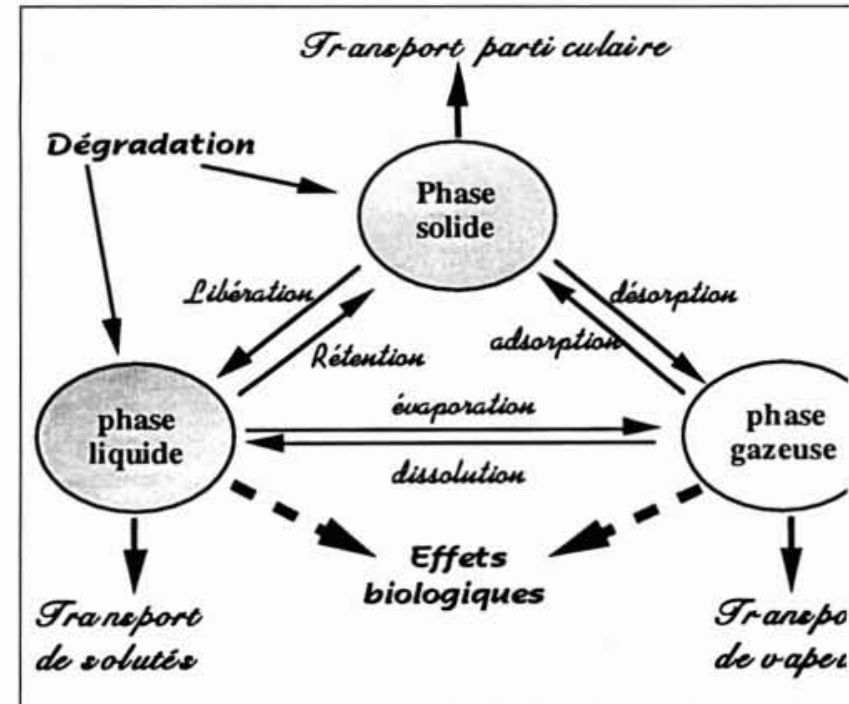

3. Phénomènes impliqués dans le devenir des pesticides dans le sol (d'après [2]).

un traitement par l'atrazine ou l'isoproturon, la libération progressive des résidus peut entretenir la pollution de l'eau pendant plusieurs années [8]. Dans le cas représenté par la figure 5 , on passe en 10 mois de 150 à $0,6 \mu \mathrm{g} \mathrm{l}^{-1}$ pour l'atrazine et de 140 à $0,5 \mu \mathrm{g} \mathrm{l}^{-1}$ pour l'isoproturon. Mais on observe par la suite une remontée de la teneur en résidus

Tableau 2 : Exportations et concentrations en herbicides dans les premières eaux de drainage d'un sol argileux et d'un sol limoneux, en fonction du délai entre le traitement et la première pluie efficace (d'après Babut et al., 1996)

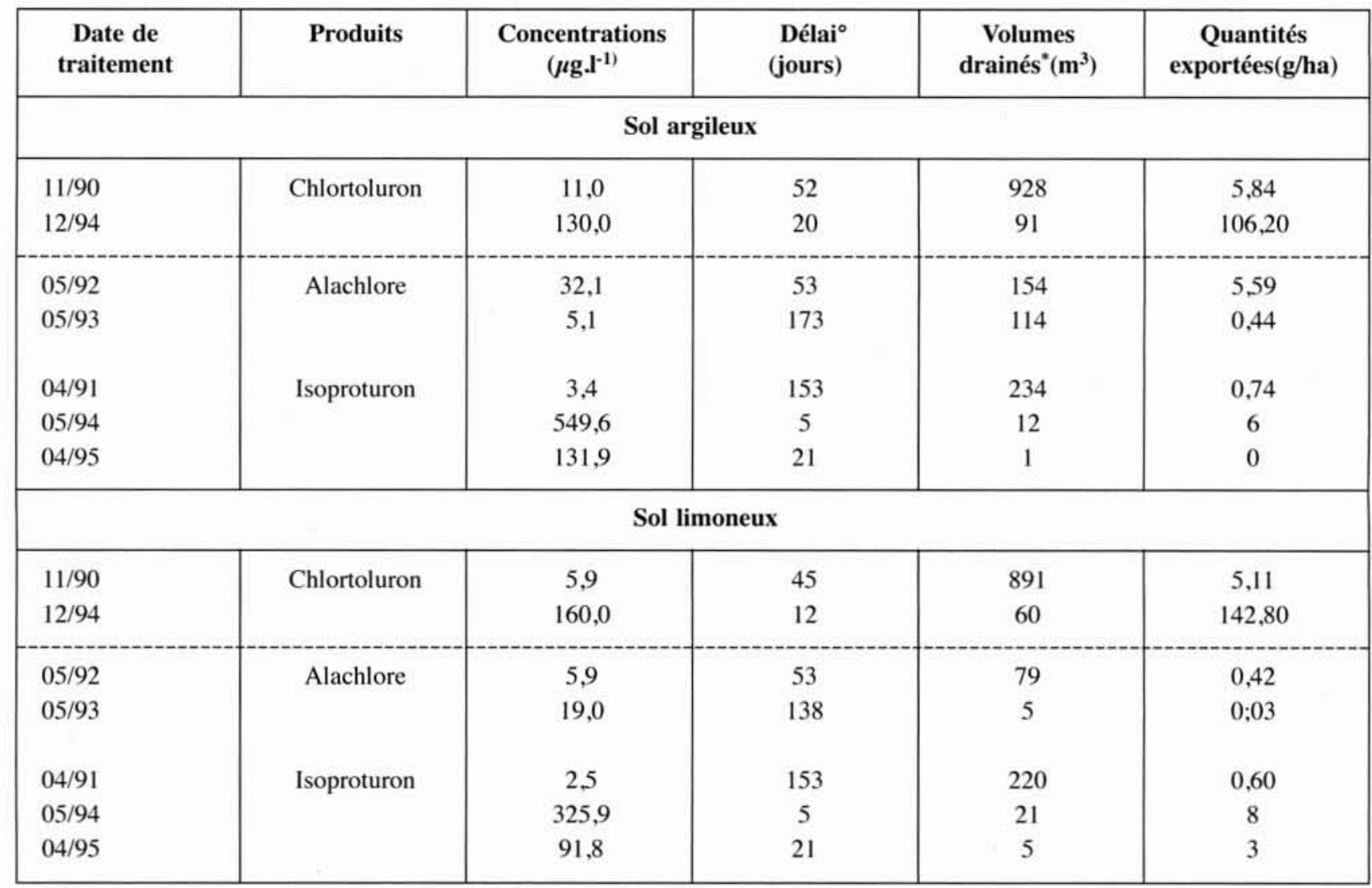

- durée entre le traitement et les premières précipitations donnant lieu à un drainage

* volume drainé au cours de cette première phase de drainage 


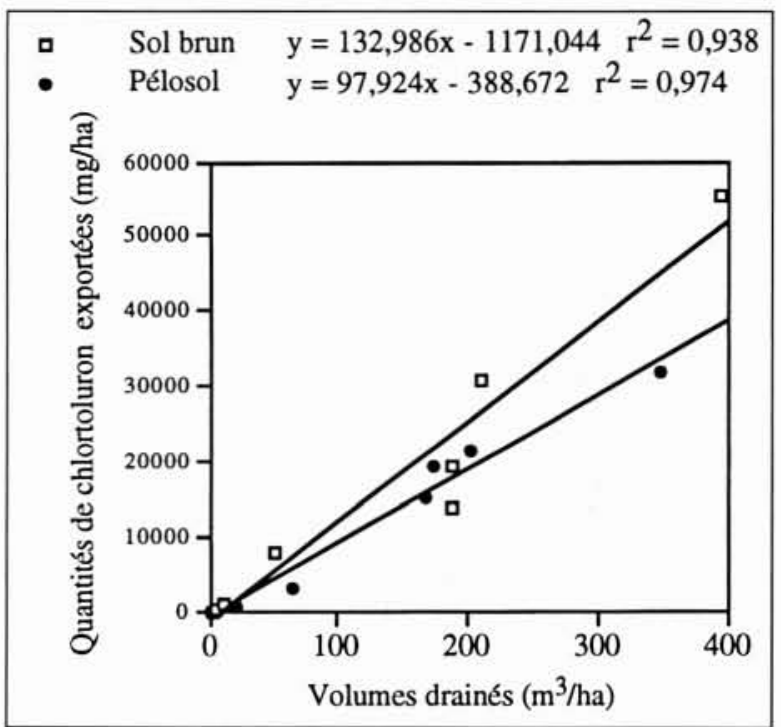

4. Corrélations entre les quantités de chlortoluron exportées au niveau des deux sols et les volumes drainés en 1994. pour chaque période estivale (mai 1996, mai 1997), vraisemblablement consécutive à des réorganisations des matières organiques $[9,10]$. Après trois ans, une teneur globale en résidus toujours supérieure à celle admise pour l'eau potable $\left(0,1 \mu \mathrm{g} \mathrm{l}^{-1}\right)$ est encore mesurée. Ces observations posent la question des conséquences sur la qualité de l'eau à moyen terme, de traitements répétés d'un même produit, comme dans le cas de l'atrazine en monoculture de maïs. Ainsi, l'effet résiduel de 20 ans de traitements successifs à l'atrazine sur monoculture de maïs (figure 6) s'est manifesté pendant huit années après arrêt de l'emploi de ce produit [8]. Mais ce laps de temps correspond en fait à une durée apparente, étroitement liée à la limite de détection de la procédure de dosage des résidus mise en œuvre.

On remarquera que la notion de pollution des eaux par les pesticides apparaît comme liée à celle des sols, mais il s'agit d'une notion relative car intimement liée aux performances analytiques et donc à la capacité de détecter ou non l'ensemble des résidus, la molécule mère et les produits issus de sa dégradation.

\section{DÉGRADATION ET NATURE DES PRODUITS DISPERSÉS}

La pollution du milieu n'est pas le simple fait des matières actives. La biodégradation des pesticides présents dans le sol est rarement de type métabolique, c'est-à-dire conduisant rapidement à leur minéralisation ou disparition totale. Même si certaines adaptations se sont mises en place localement et pour certaines molécules (carbofuran, atrazine ...), dans la majorité des cas leur dégradation relève d'un processus de co-métabolisme qui se traduit par une dégradation très lente et la production d'un grand nombre de métabolites $[12,13,14]$ dont la diversité dépend des conditions de milieu et donc vraisemblablement du type de populations microbiennes présentes. Ainsi, la biodégradation de l'isoproturon fait apparaître 2 ou 5 métabolites intermédiaires suivant le type de sol (tableau 3).

Présents dans le sol le plus souvent en quantités minimes, ces produits sont entraînés par l'eau à des concentrations trop faibles pour être détectés par les moyens classiques mis en œuvre. Ainsi, pour l'atrazine, il est clair à présent que son métabolite majeur, la dé-éthylatrazine, est plus polluant que la molécule mère, mais bien d'autres métabolites non dosés sont également présents (figure 7).

D'une manière générale, il résulte vraisemblablement de la variabilité de la biodégradation par co-métabolisme des produits phytosanitaires, une pollution de l'eau diversifiée, dont l'ampleur n'est pas encore appréciée pas plus que les effets aux niveaux toxicologique et écotoxicologique [15].
5. Evolution des teneurs en résidus d'atrazine et d'isoproturon dans les percolats de colonnes de sol, jusqu'à trois ans après le traitement. 


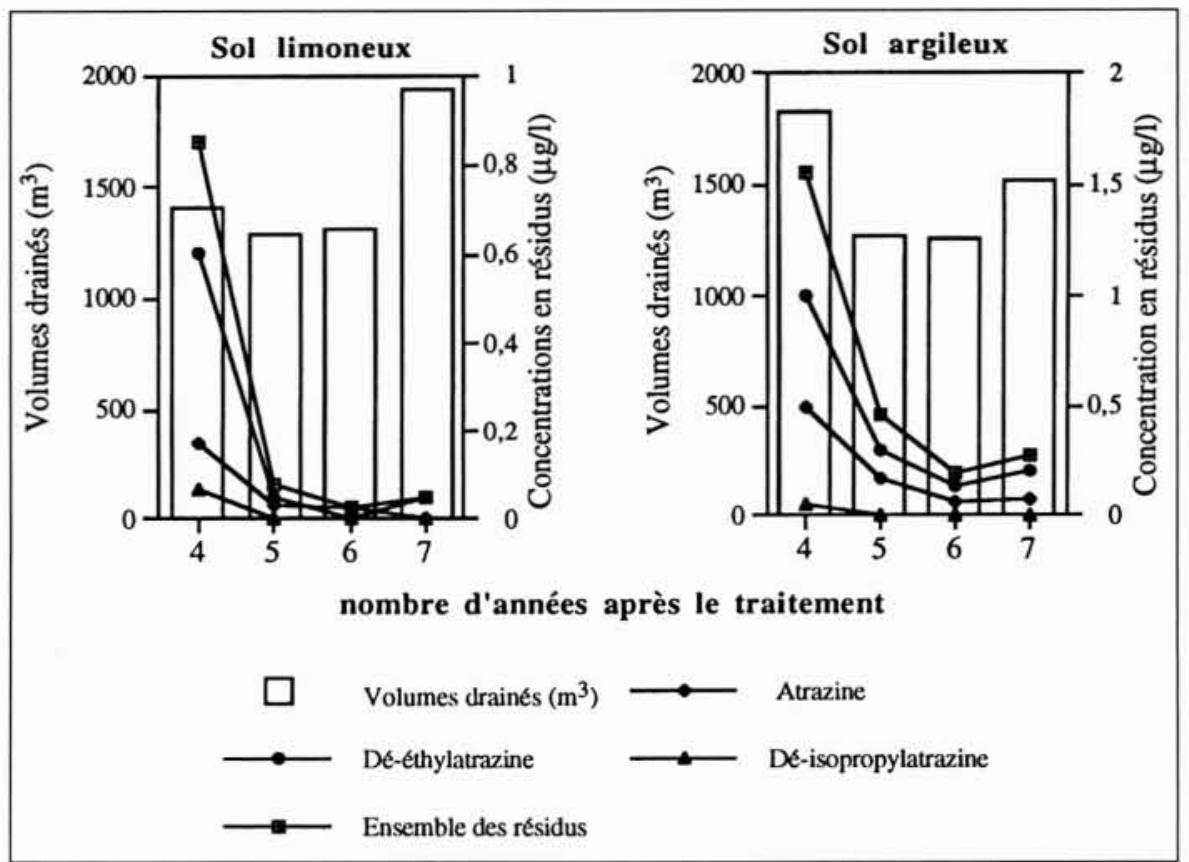

6. Evolution des concentrations d'atrazine et de ses dérivés chlorés dans les eaux de drainage, quatre à sept ans après le dernier traitement pour deux parcelles soumises aux mêmes conditions climatiques et culturales.

7. Composition des eaux de percolation de colonnes de sol traitées avec de l'atrazine marquée au ${ }^{14} \mathrm{C}$ sur le noyau en fonction des dates de prélèvement et exprimées en pourcentage de la radioactivité présente dans chaque échantillon (d'après [16])

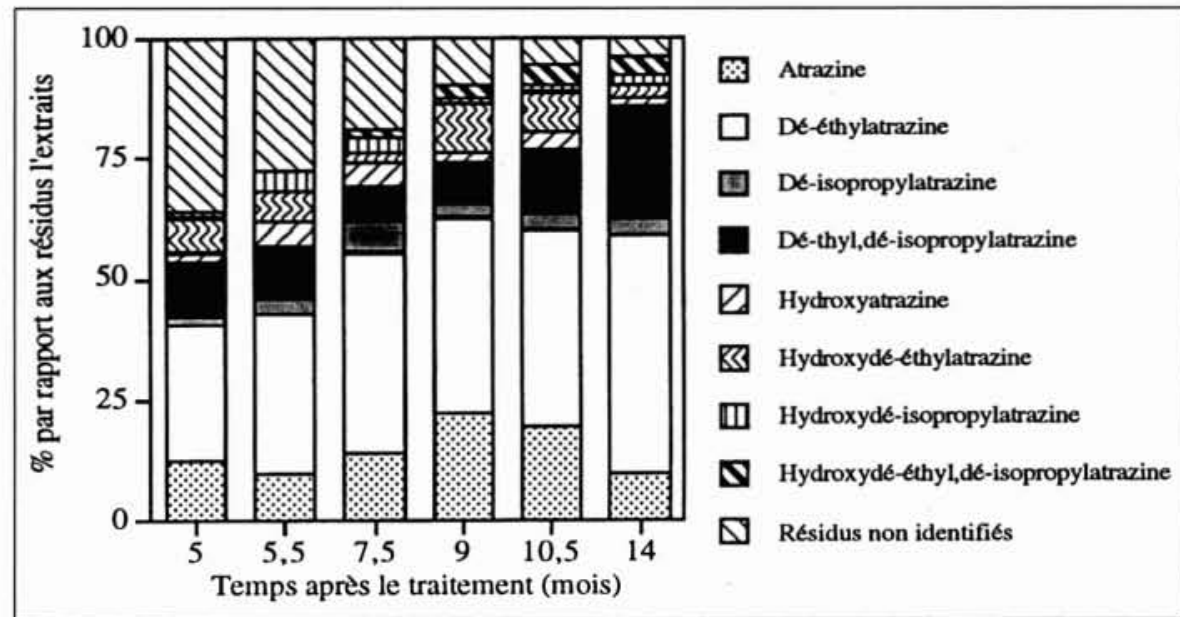

Tableau 3 : Dégradation de l'isoproturon $-{ }^{14} \mathrm{C}$ dans un sol brun calcaire et un sol brun acide (quantités de résidus extractibles exprimés en pourcentages de la radioactivité introduite au temps 0) (d'après [II]).

\begin{tabular}{|c|c|c|c|c|c|c|c|}
\hline \multicolumn{8}{|c|}{ Sol brun calcaire } \\
\hline Temps après le traitement (mois) & $\mathbf{0}$ & 0,5 & 1 & 1,5 & 2 & 3 & 4 \\
\hline \multicolumn{8}{|l|}{ Résidus extractibles (\%) } \\
\hline Isoproturon & 83,5 & 56,4 & 34,2 & 23,3 & 17,1 & 12,0 & 6.5 \\
\hline Monodéméthyl-isoproturon & 6,0 & 7,9 & 8,4 & 6,2 & 4,6 & 4,4 & 2,6 \\
\hline Monodéméthyl hydroxy-isoproturon & 0,3 & 4,0 & 6,0 & 5,3 & 4,5 & 2,5 & 1,6 \\
\hline \multicolumn{8}{|c|}{ Sol brun acide } \\
\hline Temps après le traitement (mois) & $\mathbf{0}$ & 0,5 & 1 & 1,5 & 2 & 3 & 4 \\
\hline \multicolumn{8}{|l|}{ Résidus extractibles (\%) } \\
\hline Isoproturon & 94,8 & 71,3 & 51,9 & 37,0 & 31,5 & 17,5 & 12,2 \\
\hline Monodéméthyl-isoproturon & 6,6 & 6,2 & 8,9 & 8,6 & 7,0 & 5,9 & 5,1 \\
\hline Monodéméthyl hydroxy-isoproturon & 0,8 & 2,4 & 2,6 & 1,6 & 2,6 & 1,8 & 1,6 \\
\hline Didéméthyl-isoproturon & 0,2 & 2,6 & 3,5 & 4,2 & 1,6 & 2,4 & 2,6 \\
\hline Non identifié ( $\mathrm{Tr}: 10,4 \mathrm{~min}$.) & 0 & 0 & 0,1 & 0,1 & 0,1 & 0,5 & 0,5 \\
\hline Non identifié ( $\mathrm{Tr}: 17 \mathrm{~min})$. & 0 & 0,7 & 1,6 & 6,7 & 8,6 & 8,8 & 9,2 \\
\hline
\end{tabular}




\section{IV $\square$ CONCLUSION}

L'entretien des espaces verts, des routes, des voies ferrées... et la protection des cultures conduisent à une utilisation massive de produits phytosanitaires et surtout de quelques molécules particulièrement performantes. L'emploi systématique de celles-ci ne va pas sans poser des problèmes de pollution des sols et des eaux pouvant atteindre des niveaux mesurables et dépasser les normes établies.

Mais la dispersion des produits phytosanitaires dans l'environnement sous l'action des excédents d'eau n'est pas seulement dépendante des quantités apportées. Cette dispersion dépend également d'une régulation exercée par le sol en fonction de ses propriétés bio-physico-chimiques, et faisant intervenir les possibilités de rétention et de dégradation autorisées par les propriétés spécifiques des matières actives. Une réduction de la dispersion peut être envisagée en prenant en compte les risques de précipitations à moyen terme, lors du traitement ou en modifiant les capacités de rétention et/ou de dégradation du sol (apports d'amendements organiques et augmentation du $\mathrm{pH}$ ).

Si la biodégradation d'un pesticide conduit à la formation de plusieurs métabolites récalcitrants, elle intervient non seulement sur la dynamique de transfert de la molécule mère mais également sur la nature des produits dispersés. Ainsi la biodégradation peut contribuer à réduire la pollution du milieu mais également à la diversifier.

\section{BIBLIOGRAPHIE}

[1] Lhoste J. et Grison P., 1989 - La phytopharmacie française - Chronique historique. Lavoisier Edit. 280 p.

[2] Calvet R., 1996 - Simulation numérique du devenir des pesticides dans le sol. Journées de formation Jeunes Chercheurs- Département Science du Sol INRA- Autrans 15-19 janvier 1996

[3] Guibaud G., Baudu M., Ayele J., 1998 - Etude du transfert par ruissellement et par drainage de pesticides utilisés en verger de pommiers. Colloque de I'Association Scientifique Européenne pour l'Eau et la Santé. Reims, 11-12 juin. (à paraitre)
[4] Buhler D.D., Randall G.W., Koskinen W.C., Wyse D.L., 1993 - Atrazine and Alachlor losses from subsurface tile drainage of a clay loam soil Journal of Environmental Quality, 22 (3), 583-588.

[5] Jarczyk HJ., 1987 - Untersuchungen zum Sickerverhalten von Pflanzenschutzmitteln in einer Monolithlysimeteranlage. PflanzenschutzNachrichten Bayer, 39, 49-77.

[6] Kubiak P., 1986 - Vergleichende Untersuchungen zur Ubertragbarkeit von Ergebnissen aus standardisierten Laborversuchen ung Agrarökosustemausschnitten auf die reale Feldsituation am Beispiel des Abbau- und Verlagerungsverhaltens der Herbizidwirkstoffe Metamitron und methabenzthiazuron in einer Parabraunerde. Diss. Univ. Bonn, Jül-Bericht, 2055.

17] Reiml D., Scheunert 1. \& Friedhelm K., 1989 - Leaching of conversion products of $14 \mathrm{C}$ Buturon from soil during 12 years after application. J. Agric. Food Chem.. 37, 244-248.

[8] Novak S., Portal J-M., Morel J-L., Schiavon M., 1998 - Mouvement des produits phytosanitaires dans le sol et dynamique de transfert par l'eau. C.R. Académie Agriculture. (à paraître).

[9] Demon M., Schiavon M., Portal J-M., Munier-Lamy C., 1994 - Seasonal dynamics of atrazine in three soils under outdoor conditions. Chemosphere, 28, 453-466.

[10] Hayar S., Munier-Lamy C., Chone T., Schiavon M., 1997 - Physicochemical versus microbial release of ${ }^{14} \mathrm{C}$-atrazine bound residues from a loamy clay soil incubated in laboratory microcosms. Chemosphere, 34 (12), 2683-2697.

[11] Pieuchot M., Perrin-Ganier C., Portal J-M., Schiavon M., 1996 - Study on the mineralization and degradation of isoproturon in three soils. Chemosphere, 33 (3), 467-478.

[12] Horvath R. S., 1972 - Microbial co-metabolism and the degradation of organic compounds in nature. Bacteriol. Rev, 36, 146-155.

[13] Fournier J. C., 1980 - Enumeration of the soil micro-organisms able to degrade 2,4-D by metabolism and co-metabolism. Chemosphere, 1,35-40.

[14] Deuet S., Dubourguier H. C., Wijffels P., 1995 - Successful land farming bioremediation of soils highly contaminated by coal tar residues. Abstract 5th International KfK/TNO Conf. Cont. Soils, Maastricht, The Netherlands

[15] Lardier P. A., 1987 - De la synergie entre insecticide et herbicide. Thèse INPL Nancy $185 \mathrm{p}$.

[16] Schiavon M., 1988 - Studies of the leaching of atrazine, of its chlorinated derivatives and of hydroxyatrazine from soil using ${ }^{14} \mathrm{C}$ ring-labeled compounds under outdoor conditions. Ecotoxicology and Environmental Safety $15,46-54$ 